\title{
RNA sequencing discloses the genome-wide profile of long noncoding RNAs in dilated cardiomyopathy
}

\author{
GUANGYONG HUANG $^{1 *}$, JINGWEN LIU $^{1 *}$, CHUANSHENG YANG $^{1 *}$, YOUZHANG XIANG $^{2}$, \\ YUEHAI WANG $^{1}$, JING WANG ${ }^{2}$, MIAOMIAO CAO ${ }^{1}$ and WENBO YANG ${ }^{3}$ \\ ${ }^{1}$ Department of Cardiology, Liaocheng People's Hospital of Shandong University, Liaocheng, Shandong 252000; \\ ${ }^{2}$ Shandong Institute for Endemic Disease Control, Jinan, Shandong 250014; ${ }^{3}$ Department of Cardiology, \\ Ruijin Hospital, Shanghai Jiao Tong University School of Medicine, Shanghai 200025, P.R. China
}

Received August 17, 2018; Accepted January 17, 2019

DOI: $10.3892 / \mathrm{mmr} .2019 .9937$

\begin{abstract}
Dilated cardiomyopathy (DCM) is a common type of non-ischemic cardiomyopathy, of which the underlying mechanisms have not yet been fully elucidated. Long noncoding RNAs (lncRNAs) have been reported to serve crucial physiological roles in various cardiac diseases. However, the genome-wide expression profile of lncRNAs remains to be elucidated in DCM. In the present study, a case-control study was performed to identify expression deviations in circulating lncRNAs between patients with DCM and controls by RNA sequencing. Partial dysregulated lncRNAs were validated by reverse transcription-polymerase chain reaction. Gene Ontology, Kyoto Encyclopedia of Genes and Genomes pathway, and lncRNA-messenger RNA (mRNA) co-expression network analyses were employed to probe potential functions of these dysregulated lncRNAs in DCM. Comparison between 8 DCM and 8 control samples demonstrated that there were alterations in the expression levels of 988 lncRNAs and 1,418 mRNAs in total. The dysregulated lncRNAs were found to be mainly associated with system development, organ morphogenesis and metabolic regulation in terms of 'biological processes'. Furthermore, the analysis revealed that the gap junction pathway, phagosome, and dilated and hypertrophic cardiomyopathy pathways may serve crucial roles in the development of DCM. The
\end{abstract}

Correspondence to: Dr Guangyong Huang, Department of Cardiology, Liaocheng People's Hospital of Shandong University, 67 Dongchang West Road, Liaocheng, Shandong 252000, P.R. China E-mail: 13346256309@163.com

Dr Wenbo Yang, Department of Cardiology, Ruijin Hospital, Shanghai Jiao Tong University School of Medicine, 197 Ruijin Second Road, Shanghai 200025, P.R. China

E-mail: yangwb_ywb@163.com

${ }^{*}$ Contributed equally

Key words: dilated cardiomyopathy, long noncoding RNA, messenger RNA, RNA sequencing
lncRNA-mRNA co-expression network also suggested that the target genes of the lncRNAs were different in patients with DCM as compared with those in the controls. In conclusion, the present study revealed the genome-wide profile of circulating lncRNAs in DCM by RNA sequencing, and explored the potential functions of these lncRNAs in DCM using bioinformatics analysis. These findings provide a theoretical foundation for future studies of lncRNAs in DCM.

\section{Introduction}

Dilated cardiomyopathy (DCM) is a common type of non-ischemic cardiomyopathy, which leads to progressive heart failure and sudden cardiac-associated mortality (1-3). DCM is characterized by ventricular chamber enlargement and systolic dysfunction, and has strong associations with cardiac development, organ morphology, myocardial energy metabolism and abnormal gene expression $(4,5)$. In patients with DCM, the possible causes of the condition are often ascribed to coronary artery disease, hypertension, viral infections and alcohol abuse (6). However, the exact mechanisms involved in the development of DCM remain unknown.

Long noncoding RNAs (lncRNAs) are a cluster of transcribed RNAs with a length $>200$ nucleotides, which lack protein-coding potential $(7,8)$. IncRNAs function by regulating their neighboring or distant target genes through connection with microRNAs and interaction with proteins $(9,10)$. Previous studies have verified that lncRNAs serve critical roles in the progression of cardiovascular diseases by modulating cardiac development, homeostasis and regeneration (7,11-14). Nevertheless, determining the genome-wide profile is urgently required to uncover the expression variation and potential functions of lncRNAs in the development of DCM.

In the present study, the genome-wide profile of circulating lncRNAs between patients with DCM and controls was detected by RNA sequencing, which is considered as a credible method to define lncRNA expression (15-17). Gene Ontology (GO) analysis, Kyoto Encyclopedia of Genes and Genomes (KEGG) pathway analysis and lncRNA-messenger RNA (mRNA) co-expression network analysis were performed to 
explore the potential functions of differentially expressed IncRNAs in DCM.

\section{Materials and methods}

Ethical statement. All procedures in the present study were approved by the Ethics Committee of the Liaocheng People's Hospital (Liaocheng, China). All subjects provided written informed consent prior to enrollment into the study.

Study subjects. The 8 control patients who admitted for routine testing and 8 patients with DCM underwent internal medical treatment were enrolled in the present study at Liaocheng People's Hospital between July 2015 and May 2016 (Table I). The inclusion criteria for patients with DCM were as follows: i) Left ventricular end-diastolic diameter (LVED) of $>50 \mathrm{~mm}$ (females) or $>55 \mathrm{~mm}$ (males); and ii) left ventricular ejection fraction (LVEF) of $<45 \%$ and/or left ventricular fraction shortening of $<25 \%$. Patients with clear causes of idiopathic DCM, including hypertension, coronary artery diseases, valvular disease, congenital defect, alcoholic cardiomyopathy, tachycardia-induced cardiomyopathy and peripartum cardiomyopathy, were excluded from the present study. Patients with a positive family history of DCM were also excluded. The following data were gathered for the DCM and control subjects: Sex, age, blood pressure on admission, heart rate, New York Heart Association classes (18), LVED, LVEF, N-terminal pro b-type natriuretic peptide, medical history and diagnostic testing (Table I).

Specimen collection. Blood samples for RNA sequencing were collected from 8 typical patients with DCM and 8 healthy controls. In addition, blood samples from another 20 patients with DCM (16 males and 4 females, $43.2 \pm 8.7$ years old) and 20 healthy controls ( 16 males and 4 females, $45.3 \pm 7.9$ years old) were collected to identify the expression levels of the top 10 lncRNAs with the highest degree via Reverse transcription-polymerase chain reaction (RT-PCR), which was conducted as described below. All the patients were diagnosed with DCM by two experienced clinicians and underwent internal medical treatment at Liaocheng People's Hospital based on the American Heart Association guidelines $(19,20)$. Peripheral venous blood $(5 \mathrm{ml})$ samples were collected from all participants in sterile tubes with ethylenediaminetetraacetic acid.

RNA isolation, library construction and sequencing. Total RNA was isolated and enriched using TRIzol ${ }^{\circledR}$ reagent (cat. no. 15596; Invitrogen; Thermo Fisher Scientific, Inc., Waltham, MA, USA). Next, ribosomal RNA was removed using the Ribo-Zero rRNA Removal kit (cat. no. MRZB12424; Illumina, Inc., San Diego, CA, USA). Ribosomal RNA-depleted RNAs were used to construct RNA libraries with the TruSeq Stranded Total RNA Library Prep kit (cat. no. RS-122-2301; Illumina, Inc.). Libraries were controlled for quality and quantified using the Agilent BioAnalyzer 2100 system (cat. no. 5067-1505; Agilent Technologies Inc., Santa Clara, CA, USA). Libraries (10 pM) were denatured as single-stranded DNA molecules, captured on Illumina flow cells, amplified in situ as clusters and finally sequenced for 150 cycles on the Illumina HiSeq sequencer (HiSeq 4000; Illumina, Inc.). All these procedures were conducted according to the manufacturer's protocol.

Analysis of RNA sequencing data. Paired-end reads were harvested from Illumina HiSeq sequencer and quality controlled by $\mathrm{Q} 30 \quad(\mathrm{P}<0.001)$. Subsequent to removing the 3 ' adaptor-trimming and low-quality reads with cutadapt software (version 1.9.3, https://cutadapt. readthedocs.io/en/stable/index.html), the high-quality trimmed reads were aligned to the reference genome (UCSC hg19) guided by the Ensembl Gff gene annotation file with HISAT2 software (version 2.0.4, http://ccb.jhu. edu/software/hisat2/index.shtml). The cuffdiff software (version 2.2.1; part of Cufflinks, http://cole-trapnell-lab. github.io/cufflinks/cuffdiff/index.html) was then used to obtain the expression profiles of lncRNAs and mRNAs in terms of the fragments per kilobase of transcript per million fragments mapped (FPKM). Next, the fold-change (FC) and P-values were calculated based on the FPKM, and differentially expressed lncRNAs and mRNAs were identified between the DCM and control groups. The criteria were set as an FC of $\geq 2$ or $\leq-2$, and a P-value of $<0.05$ between two groups. P-values were corrected with the false discovery rate method. Hierarchical clustering with average linkage was used to calculate the distinguishable expression patterns. RNA sequencing data were uploaded to the Gene Expression Omnibus database (accession no. GSE101585; https:/www. ncbi.nlm.nih.gov/geo/query/acc.cgi?\&acc=GSE101585).

$R T-P C R$. Total RNA from the blood samples was isolated and enriched with TRIzol ${ }^{\circledR}$ reagent (Thermo Fisher Scientific, Inc.), and then the concentration was measured with a NanoDrop ND-1000 spectrophotometer (Nanodrop Technologies; Thermo Fisher Scientific, Inc., Wilmington, MA, USA). Total RNA was reverse transcribed into cDNA with the SuperScript III reverse transcriptase (cat. no. 18080044; Invitrogen; Thermo Fisher Scientific, Inc.). Subsequently, 2X PCR master mix (CloudSeq Biotech, Inc., Shanghai, China) was applied to perform the PCR with the target RNAs and internal reference, $\beta$-actin (ACTB) of the samples. The primers were as follows: TCONS_00028585, forward: 5'-CGC AGAAGCACAGGGTTAAT-3', reverse, 5'-GCTGTAGCT TGCTGGCTCTT-3'; ENST00000439796, forward, 5'-TGA AAAACACCGCAGTCAAA-3', reverse, 5'-AGGCCCTAA AGAAGGCCATA-3'; TCONS_12_00009351, forward, 5'-ATGAAGCTGGGCAAATCAGT-3', reverse, 5'-AGATGG CACAAATGCTCTCC-3'; ENST00000448134, forward 5'-TTGGTGGACGGAACAGTGTA-3', reverse, 5'-GAGTTA GCCAGTGGGGTGAA-3'; ENST00000607876, forward, 5'-CCCTCACACCTCTGTGTCCT-3', reverse, 5'-GTGACA CAGCCACAGTCCAC-3'; and ACTB, forward, 5'-GTGGCC GAGGACTTTGATTG-3' and reverse, 5'-CCTGTAACAACG CATCTCATATT-3'.

The thermal cycling conditions were set to 40 cycles at $95^{\circ} \mathrm{C}$ for $10 \mathrm{sec}$ and $60^{\circ} \mathrm{C}$ for $60 \mathrm{sec}$, following predegeneration for $10 \mathrm{~min}$ at $95^{\circ} \mathrm{C}$. Data were analyzed using the $2^{-\Delta \Delta \mathrm{Cq}}$ method $(21,22)$. 
Functional group analysis. The cis target genes were analyzed through the gene annotations provided by the University of California (Oakland, CA, USA) (23), and trans target genes were predicted using BLAT (24). GO analysis, which contained three domains, namely the 'biological process', 'cellular component' and 'molecular function', was performed to explore the functions of differentially expressed coding genes with the Database for Annotation, Visualization and Integrated Discovery (http://david.abcc.ncifcrf.gov) (25). Pathway analysis was also adopted to explore potential pathways of differentially expressed coding genes, according to KEGG pathways (https://www.genome.jp/kegg/). The criteria were set as follows: Fold-change of $\geq 2$ or $\leq-2$, and P-value of $<0.05$ between the DCM and control groups.

Analysis of the lncRNA-mRNA co-expression network. Pearson's correlation coefficient (PCC) was calculated for each of the IncRNA-mRNA pairs across the DCM and control groups. Co-expressed lncRNA-mRNA pairs with $\mathrm{PCC}>0.99$ and $\mathrm{P}<0.01$ were selected. In total, two lncRNA-mRNA co-expression networks were initially constructed based on co-expressed lncRNA-mRNA pairs in the DCM and control groups. The 'lost' network presents the lncRNA-mRNA co-expression pairs, which only appeared in the control group, and the 'obtained' network presents the co-expression pairs, which only appeared in the DCM group. Finally, the dynamic lncRNA-mRNA co-expression network was constructed by combining the 'lost' and 'obtained' networks. Subsequently, key lncRNA nodes were identified in the combined network. According to the mRNAs paired with key lncRNAs, GO analysis was performed to separately identify the significantly enriched biological processes in the DCM and the normal co-expression networks. $\mathrm{P}<0.05$ was considered to indicate a statistically significant difference.

\section{Results}

Overview of IncRNAs between patients with DCM and controls determined by RNA sequencing. RNA sequencing detected a total of 88,768 lncRNAs in the serum of DCM and control subjects. In order to assess the differentially expressed lncRNAs, the criteria were set as fold-change of $\geq 2$ or $\leq-2$, and P-value of $<0.05$ between the DCM and control groups. A hierarchical clustering approach was adopted to verify the consistency in the specimens, according to dysregulated lncRNAs (Fig. 1A). A heat map of partially dysregulated lncRNAs is presented in Fig. 1B. Compared with the control group, 998 dysregulated lncRNAs were identified in patients with DCM, including 661 unregulated lncRNAs and 327 downregulated lncRNAs (Fig. 1C). In addition, a scatter plot was implemented to identify differentially expressed lncRNAs between DCM and control subjects (Fig. 1D).

Distribution profiles of differentially expressed lncRNAs. The dysregulated lncRNAs were subsequently classified in five categories based on their genomic locations, which included intergenic $(n=654)$, bidirectional $(n=220)$, exonic $(n=25)$, antisense $(n=6)$ and intronic $(n=5)$ lncRNAs (Fig. 2A). In terms 


\section{A}

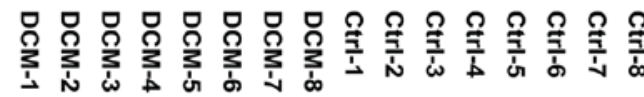

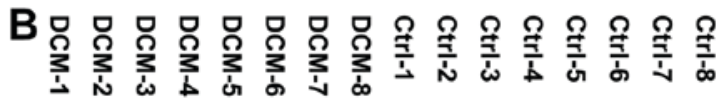
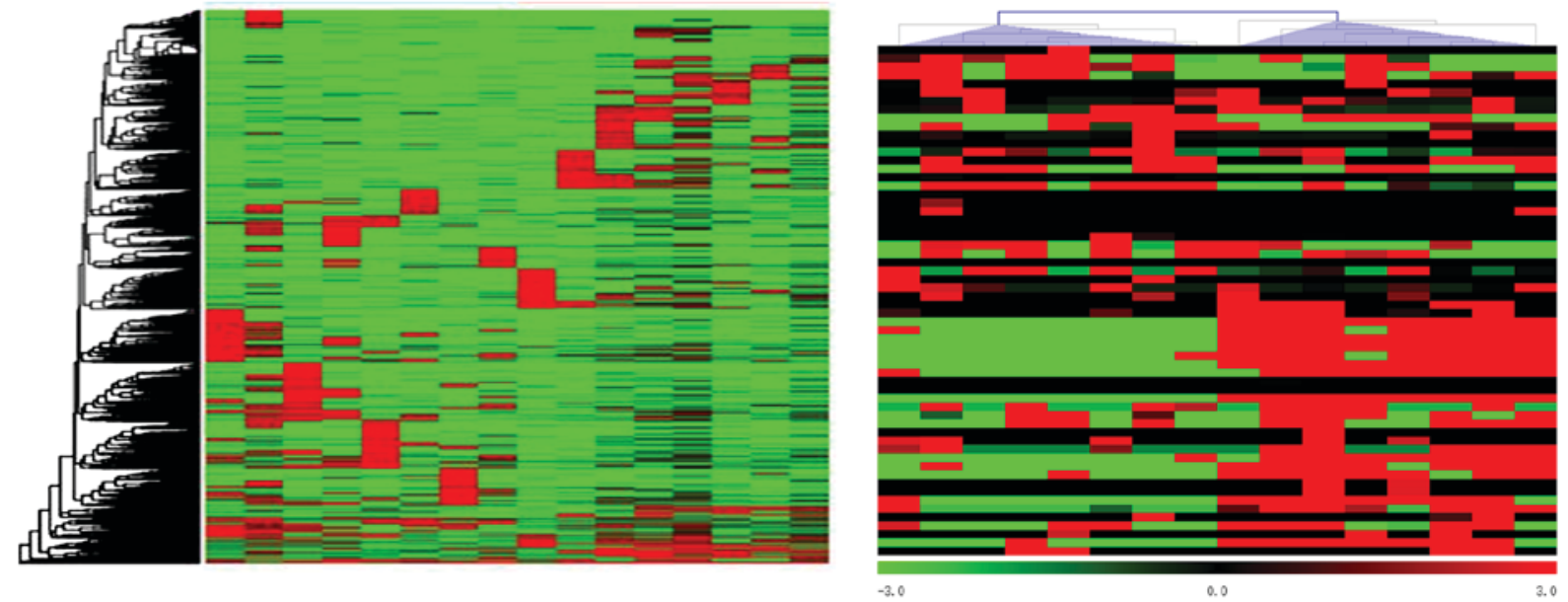

C

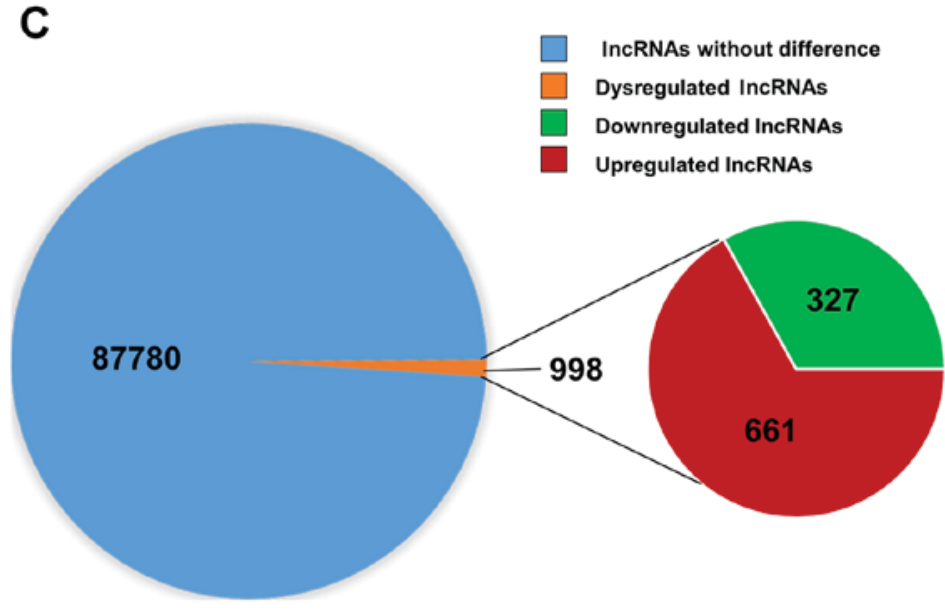

D

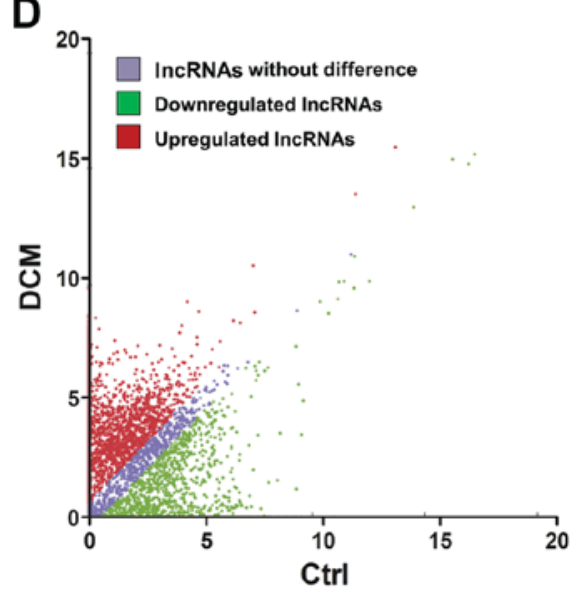

Figure 1. Overview of lncRNAs differentially expressed between 8 patients with DCM and 8 controls, detected by RNA sequencing. (A) Hierarchical clustering of partial differentially expressed lncRNAs. (B) Heat map of partially dysregulated lncRNAs. (C) A total of 88,768 lncRNAs were detected by RNA sequencing. Among these, 988 lncRNAs were differentially expressed between patients with DCM and controls, including 661 upregulated and 327 downregulated IncRNAs. (D) A scatter plot of the total lncRNAs between patients with DCM and controls. The criteria used included a fold-change of $\geq 2$ or $\leq-2$, and a P-value of $<0.05$. Ctrl, control; DCM, dilated cardiomyopathy; lncRNA, long noncoding RNA.

of length distribution, dysregulated lncRNAs ranged mainly between 500 and 10,000 base pairs (bp; Fig. 2B). These differentially expressed IncRNAs were scattered throughout the human chromosomes (Fig. 2C).

RT-PCR validation of differentially expressed lncRNAs. The top 10 upregulated IncRNAs presenting the highest fold-change were TCONS_00028585, ENST00000439796, TCONS_ 12_00009351, ENST00000448134, ENST00000607876, TCONS_00015639,ENST00000489016, TCONS_00011165, ENST00000322227 and ENST00000458361. The top 10 downregulated 1 ncRNAs presenting the highest fold-change were TCONS_00003609, TCONS_00018640, ENST00000606664, uc003uey.1, ENST00000494231, EN S T 00000419628 , ENST 00000435049 , ENST00000609755, TCONS_00026839 and ENST00 000500180 (Fig. 3A). Next, five dysregulated lncR NAs were selected to confirm the IncRNA expression, including TCONS_00028585 (Fig. 3B), ENST00000439796 (Fig. 3C), TCONS_12_00009351 (Fig. 3D), ENST00000448134
(Fig. 3E) and ENST00000607876 (Fig. 3F). The results verified that the expression levels of these IncRNAs were significantly upregulated in DCM patients as compared with those in control subjects.

GO and KEGG pathway analyses of dysregulated lncRNAs. In order to assess the potential functions of these dysregulated lncRNAs, GO and KEGG pathway analyses were performed with the target coding genes of downregulated lncRNAs and upregulated IncRNAs, separately. GO analysis was comprised of three networks, including the 'biological process', 'cellular component' and 'molecular function'. Through GO analysis of neighbor coding genes, the downregulated lncRNAs were found to be mostly enriched as follows: System development, anatomical structure development and cellular component organization in the 'biological process' analysis (Fig. 4A); organelle, intracellular organelle and intracellular part in the 'cellular component' analysis (Fig. 4B); and protein binding, cytoskeletal protein binding and enzyme binding in the 'molecular function' analysis (Fig. 4C). KEGG pathway 


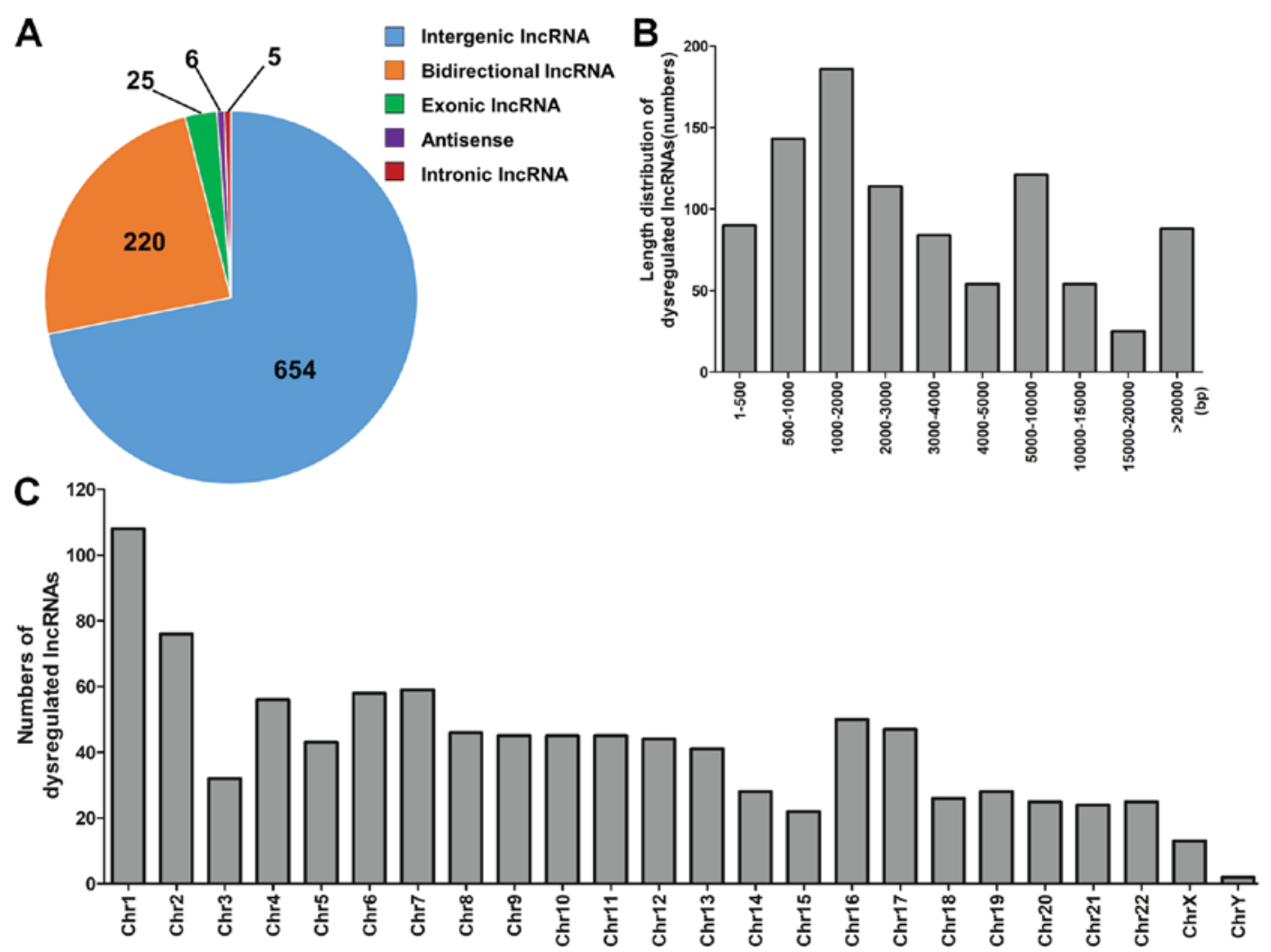

Figure 2. Distribution profiles of dysregulated lncRNAs. (A) Class distribution of dysregulated lncRNAs, including 654 intergenic, 220 bidirectional, 25 exonic, 6 antisense and 5 intronic lncRNAs. (B) Length distribution of dysregulated lncRNAs, which were mainly between 500 and 10,000 bp in length. (C) Chromosomal distribution of dysregulated lncRNAs. bp, base pairs; Chr, chromosome; lncRNA, long noncoding RNA.

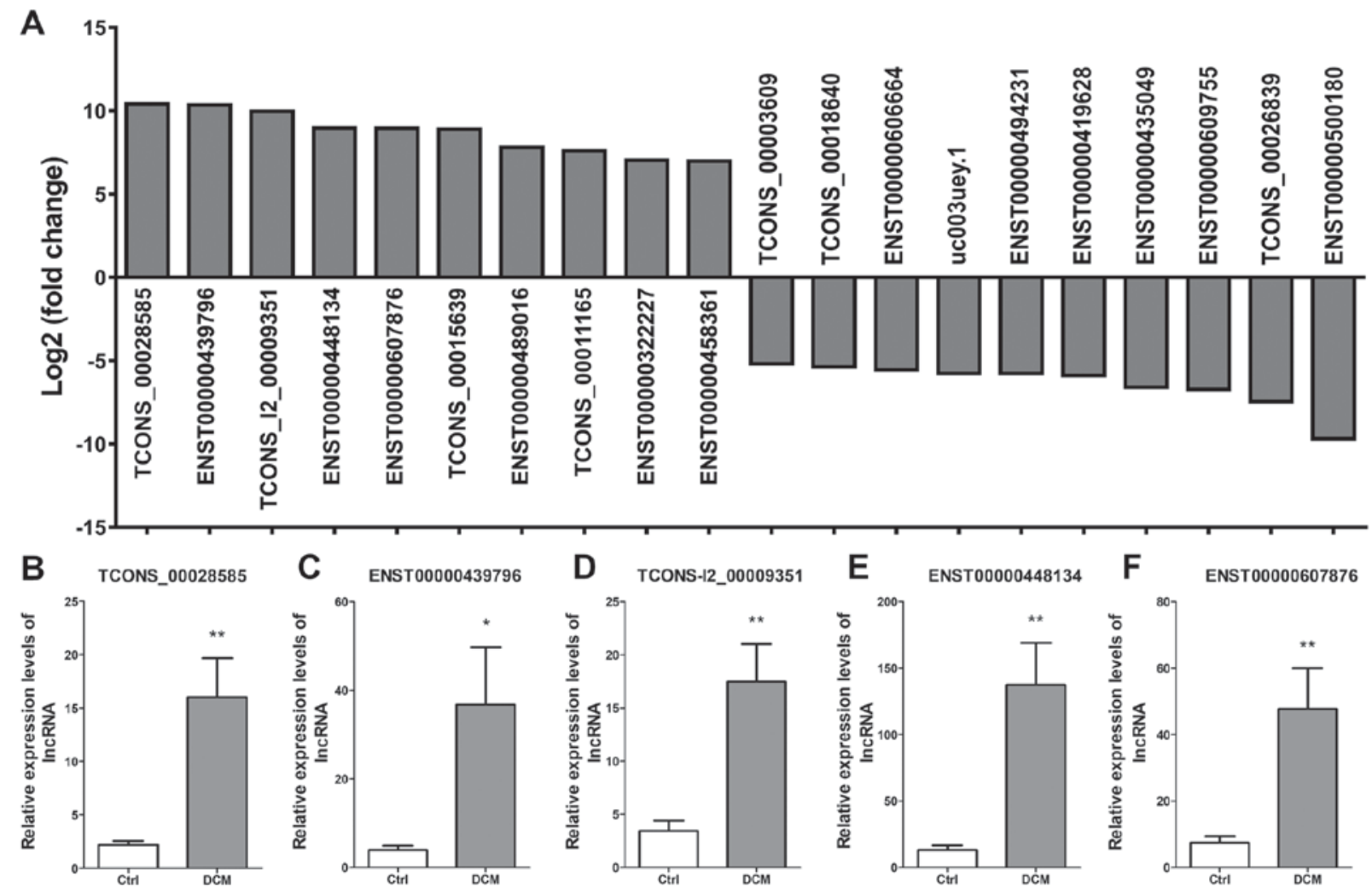

Figure 3. Partial dysregulated lncRNAs and validation of the results of RNA sequencing. (A) Top 10 upregulated and top 10 downregulated lncRNAs with the highest fold-change between patients with DCM $(\mathrm{n}=8)$ and controls $(\mathrm{n}=8)$. Reverse transcription-polymerase chain reaction validation of five differentially expressed lncRNAs was conducted, including (B) TCONS_00028585, (C) ENST00000439796, (D) TCONS_12_00009351, (E) ENST00000448134 and (F) ENST00000607876. The experiments were repeated for three times. "P $<0.05$ and ${ }^{* *} \mathrm{P}<0.01$, vs. Ctrl group. IncRNA, long noncoding RNA; DCM, dilated cardiomyopathy; Ctrl, control. 


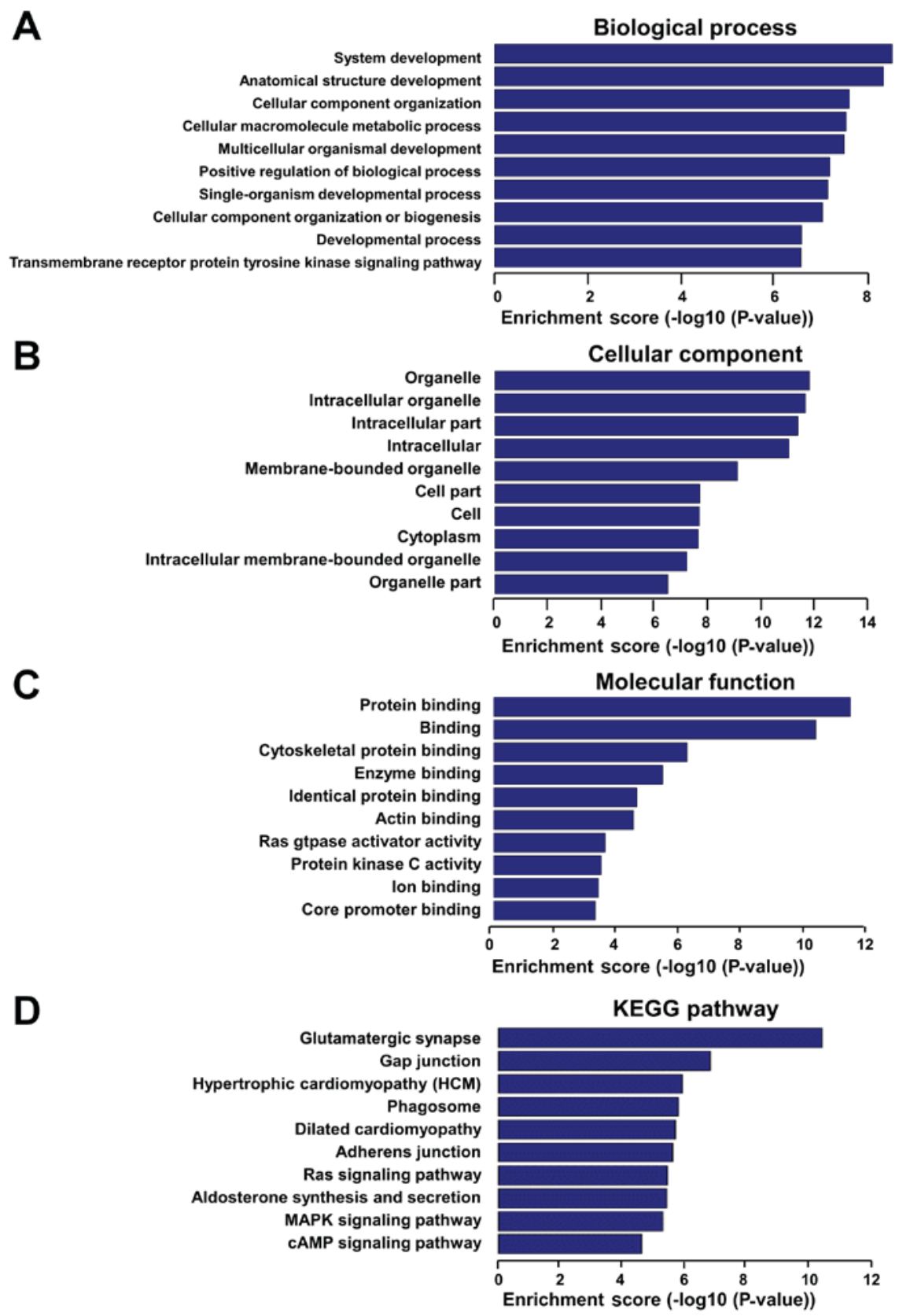

Figure 4. GO and KEGG pathway analyses were performed for protein-coding neighbors of downregulated lncRNAs. GO analysis results of downregulated lncRNAs according to (A) 'biological process', (B) 'cellular component' and (C) 'molecular function'. (D) Pathway analysis results of downregulated lncRNAs based on the KEGG database. GO, Gene Ontology; KEGG, Kyoto Encyclopedia of Genes and Genomes; lncRNA, long noncoding RNA.

analysis revealed that downregulated lncRNAs were mainly associated with gap junction, hypertrophic cardiomyopathy, phagosomes and DCM pathways (Fig. 4D). In addition, upregulated lncRNAs were mainly associated with the regulation of metabolic process, organ morphogenesis and regulation of nucleobase-containing compound metabolic process in the biological process analysis (Fig. 5A), with the cell projection, cell junction and intracellular part in the 'cellular component' analysis (Fig. 5B), and with protein binding, enzyme binding and calcium ion transmembrane transporter activity in the 'molecular function' analysis (Fig. 5C). The upregulated lncRNAs exhibited a strong association with hypertrophic cardiomyopathy and various cancer pathways, according to KEGG pathway analysis (Fig. 5D).
Overview of mRNAs between patients with DCM and controls determined by RNA sequencing. By RNA sequencing, a total of 1,418 differentially expressed mRNAs (800 upregulated mRNAs and 618 downregulated mRNAs) were identified among the 20,315 detected mRNAs between the DCM and control groups (Fig. 6A), according to the criteria of a fold-change of $\geq 2$ or $\leq-2$, and $\mathrm{P}<0.05$. A scatter plot was implemented to identify the differentially expressed mRNAs (Fig. 6B). The top 10 upregulated mRNAs were DCAF15, DEPDC4, RASA4, ZNF227, ZNF559-ZNF177, POLR1C, TES, KCNIP2, RAD52 and CD300LF. The top 10 downregulated mRNAs were HLA-A, PHB, SNRPN, DPEP2, OR6S1, ZNF233, DUT, ACD, KIAA1324 and C11orf48 (Fig. 6C). 
A

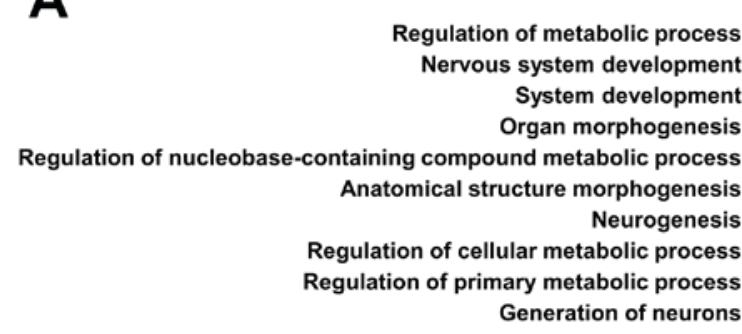

B

C

D

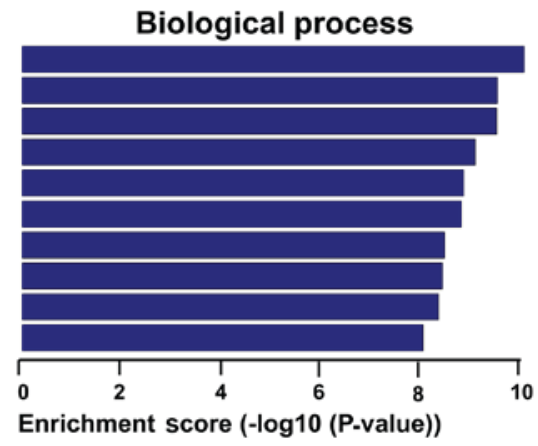

Cellular component

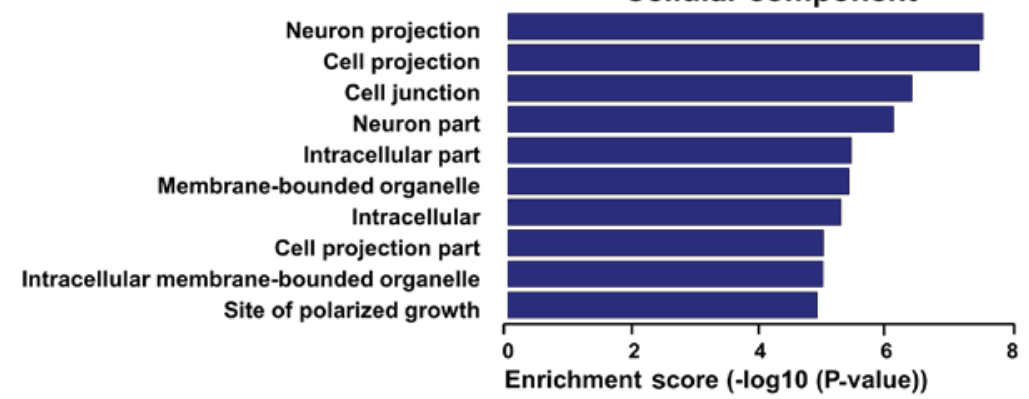

Molecular function

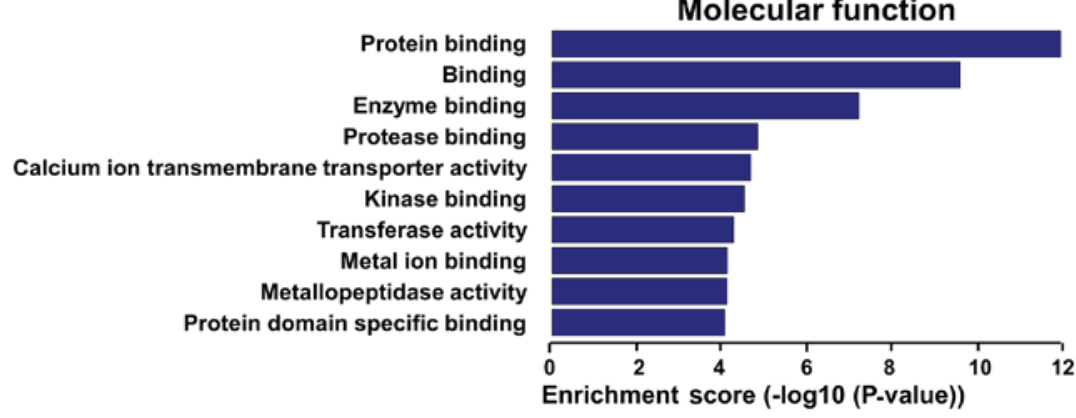

KEGG pathway

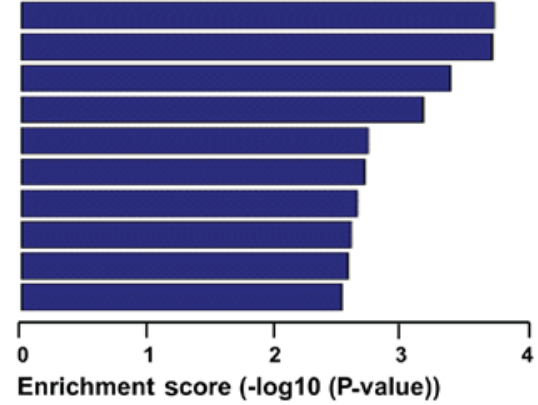

Figure 5. GO and KEGG pathway analyses were performed for protein-coding neighbors of upregulated lncRNAs. GO analysis results of upregulated lncRNAs according to (A) 'biological process', (B) 'cellular component' and (C) 'molecular function'. (D) Pathway analysis results of upregulated lncRNAs based on the KEGG database. GO, Gene Ontology; KEGG, Kyoto Encyclopedia of Genes and Genomes; lncRNA, long noncoding RNA.

lncRNA-mRNA co-expression network analysis. A dynamic lncRNA-mRNA co-expression network was constructed based on the PCC to identify the correlation between differentially expressed lncRNAs and mRNAs. Initially, the DCM and control lncRNA-mRNA co-expression networks were constructed individually. A total of 1,331 lncRNA-mRNA pairs were detected in the DCM co-expression network ('obtained' network), while 1,348 lncRNA-mRNA pairs were identified in the control co-expression network ('lost' network). Next, the two networks were combined into a total co-expression network containing 676 nodes (69 lncRNA nodes and 607 mRNA nodes) and 2,661 edges (Fig. 7). The top 10 lncRNA nodes (hub nodes) with the highest degree were XLOC_004581, XLOC_12_010969,ENSG00000202198,ENSG00000251408, XLOC_008043, BC015774, ENSG00000267172, AK055324, AK094644 and CEP83-AS1 (Fig. 8A).

Furthermore, the blood samples from another 20 patients with DCM and 20 healthy controls were collected to identify the expression levels of the top 10 lncRNA nodes with the highest degree using RT-PCR assay (Fig. 8B). According to the GO analysis, the top 10 lncRNA-mRNA co-expression pairs with the highest degree were mainly associated with 
A
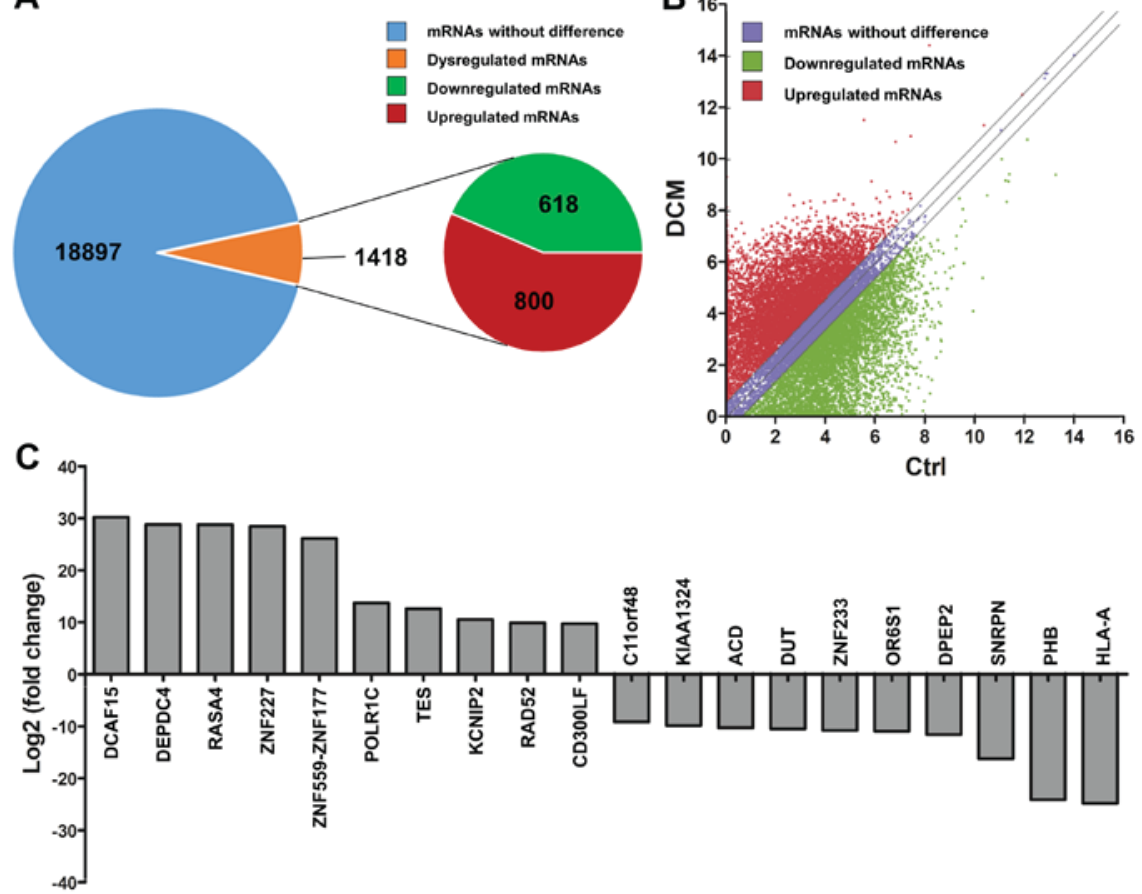

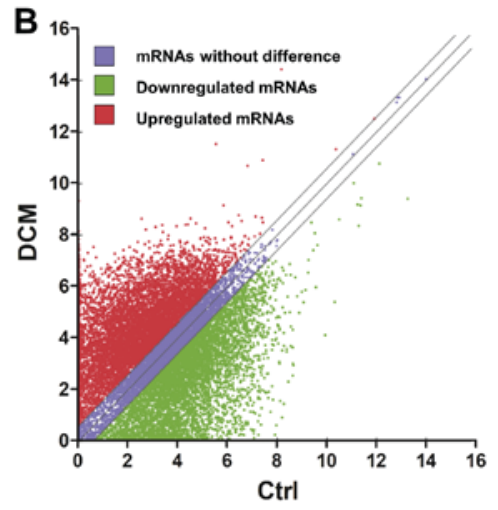

B

. 
A

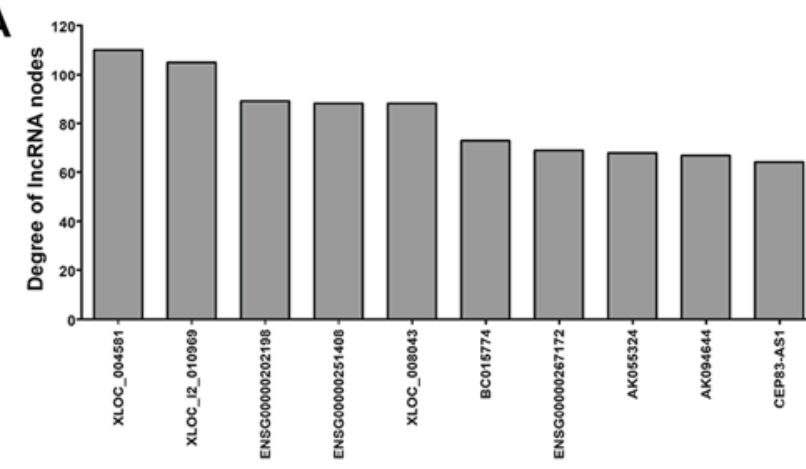

B

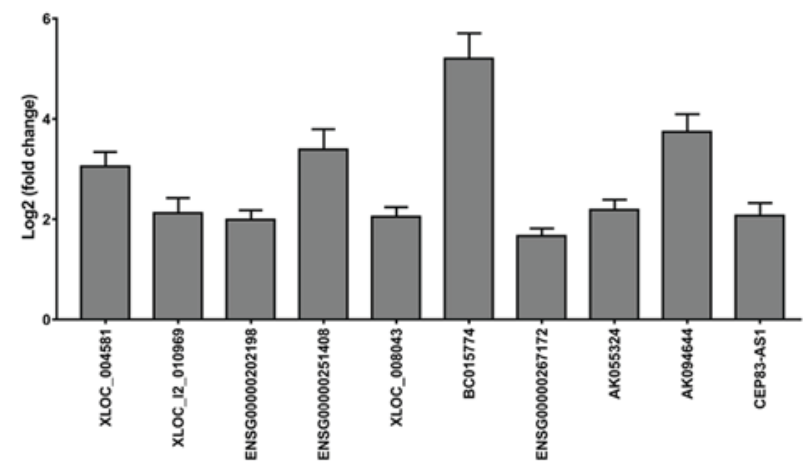

C

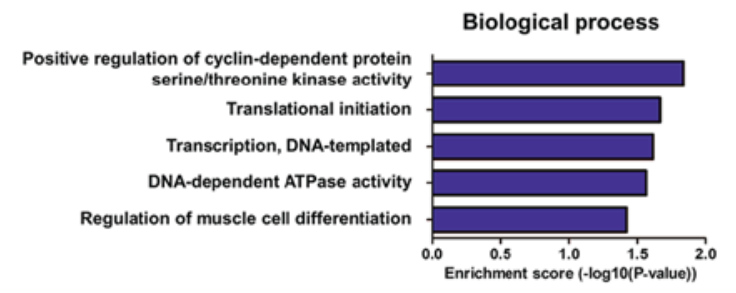

D

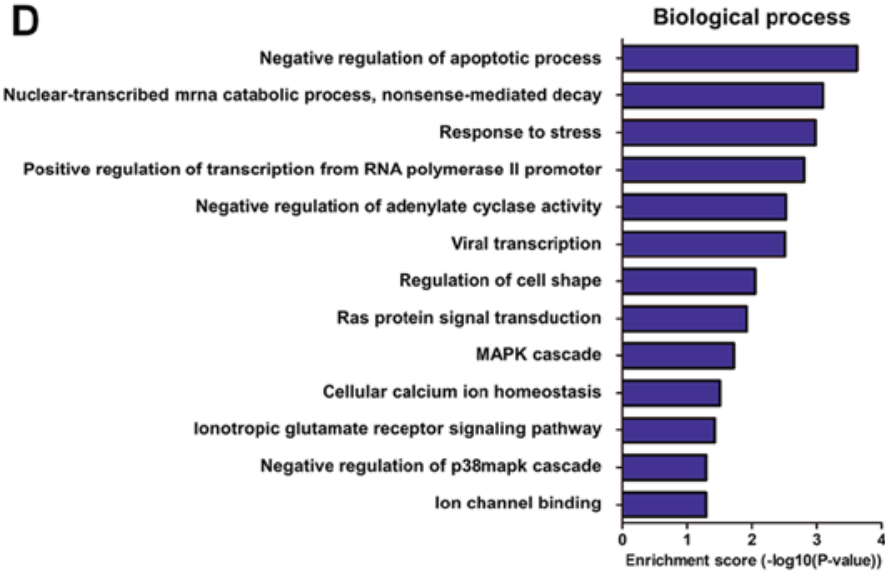

Figure 8. Analysis of IncRNA-mRNA co-expression network between patients with DCM and controls. (A) Top 10 lncRNA nodes with the highest degree. (B) Reverse transcription-polymerase chain reaction assay of blood samples from another 20 patients with DCM and 20 healthy controls, used to identify the expression levels of the top 10 lncRNAs with the highest degree. The experiments were repeated for three times. Biological processes associated with the top 10 lncRNA-mRNA co-expression pairs in the (C) DCM and (D) control groups are presented. DCM, dilated cardiomyopathy; lncRNA, long noncoding RNA; mRNA, messenger RNA.

phagosome, and dilated and hypertrophic cardiomyopathy pathways, may serve crucial roles in the genesis and development of DCM. Through the biological analysis of the top 10 IncRNA-mRNA pairs with the highest degree in the dynamic lncRNA-mRNA co-expression network, it was observed that the top 10 lncRNAs were mainly associated with DNA-templated transcription, translational initiation and regulation of muscle cell differentiation in the DCM group. However, these top 10 lncRNAs were associated with the negative regulation of apoptotic process, nuclear-transcribed mRNA catabolic process and regulation of response to stress in the control group.

Due to the severe outcome of patients with DCM, it is imperative to uncover effective biomarkers and therapy targets for the diagnosis and treatment of this disease. IncRNAs are implicated in diverse biological processes in different diseases, such as in cell-cycle control, differentiation, apoptosis, chromatin remodeling, maintenance of the nuclear structure integrity, and transcriptional and post-transcriptional processing (26-29). Recent studies have demonstrated that lncRNAs serve critical roles in cardiac development, and participate in cardiac homeostasis and regeneration as epigenetic regulators of cardiac gene expression $(11,13,30)$. Circulating lncRNAs have also been reported to be involved in the pathophysiology of cardiovascular diseases. For instance, the circulating lncRNA HOTAIR performed a protective role in acute myocardial infarction via the negative regulation of
miR-1 (31). Although a few pathogenic lncRNAs have been reported in DCM (32-34), the majority of these studies did not employ RNA sequencing tools and bioinformatics analysis; therefore, more comprehensive information remains to be elucidated. In the present study, the genome-wide profile of lncRNAs was identified by comparing their expression in the serum of patients with DCM and controls by RNA sequencing. The present study identified the IncRNA variation in DCM and provides a critical insight on the potential mechanisms underlying this disease. In addition, the present results are important for the identification of novel biomarkers of idiopathic DCM.

Cardiac sarcomere proteins are vitally important in maintaining a normal cardiac structure. Mutations of the genes encoding these proteins, particularly the titin and myosin heavy chain 7 genes, may result in the occurrence of DCM (35-38). In the present study, it was observed that the titin gene was regulated by several lncRNAs, including ENGS00000237298, ENGS00000267784,ENGS00000442329,ENGS00000603415 and ENGS00000603521. In addition, the myosin heavy chain 7 gene was regulated by NR126491. These results suggested the potential regulatory mechanisms of these sarcomere proteins, although mutations in sarcomeric genes were not examined in the present study. Furthermore, more potential genes were identified at the genome-wide level, which may function as key regulators in the development of DCM.

The IncRNA-mRNA co-expression network analysis was performed by combining IncRNA-mRNA pairs 


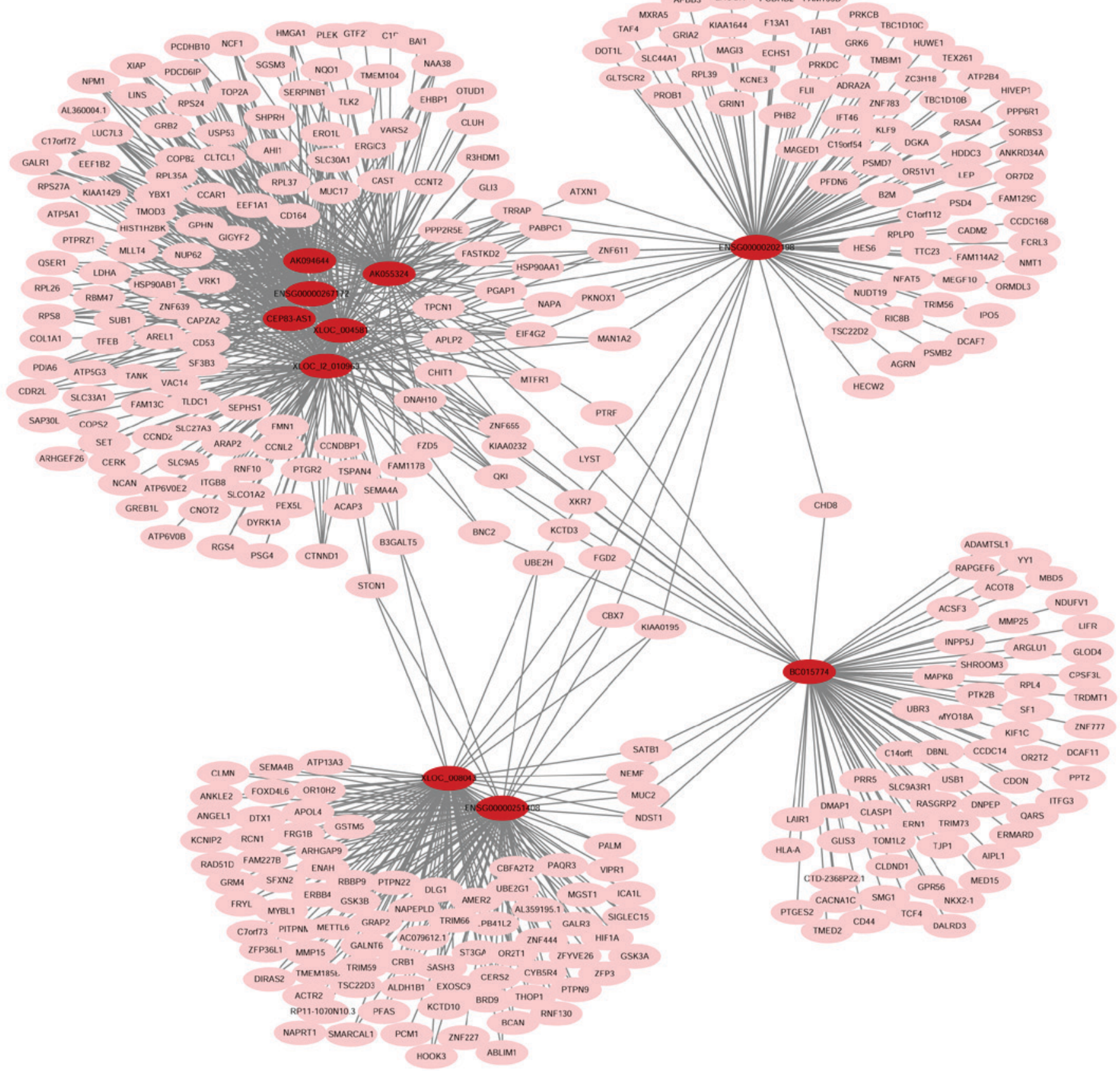

Figure 9. Co-expression network with the top 10 lncRNA nodes with the highest degree in the dynamic lncRNA-mRNA co-expression network. Target mRNAs of the top 10 lncRNAs with the highest degrees are shown in the lncRNA-mRNA co-expression network. The red nodes represent lncRNAs, and the pink nodes represent mRNAs. IncRNA, long noncoding RNA; mRNA, messenger RNA.

identified separately in the DCM and control groups. Biological processes were analyzed with the target mRNAs of the top 10 lncRNA nodes with the highest degree in the DCM and control groups. The function of the majority of these lncRNAs remain unknown, apart from ENSG00000202198 (also known as RN7SK), which was identified to be associated with immunodeficiency syndrome (39). Notably, these 10 lncRNAs participated in different biological processes between the DCM and control groups. It is known that lncRNAs are able to regulate multigene expression to participate in biological processes $(9,10)$. The findings of the present study suggested that lncRNAs were also able to change their main target genes, according to different disease circumstances. Modifying the cardiac circumstances of patients with DCM may be an effective therapy to relieve the progression of this disease.

In conclusion, the present study identified the genome-wide profile of IncRNAs in DCM by RNA sequencing. Bioinformatics analysis identified the potential functions of these lncRNAs. The IncRNA-mRNA co-expression network analysis revealed that the main target genes were different in DCM. Although the study mainly focused on bioinformatics analysis without experimental confirmation of the pathophysiology in DCM, the findings offered a novel insight into the disease pathogenesis and provided a theoretical basis for future studies of lncRNAs in DCM. 


\section{Acknowledgements}

Not applicable.

\section{Funding}

The present study was supported by the National Natural Science Foundation of China (grant no. 81573095).

\section{Availability of data and materials}

The datasets generated and/or analyzed during the current study are available in the Gene Expression Omnibus database (accession no. GSE101585; https://www.ncbi.nlm.nih. gov/geo/query/acc.cgi?\&acc=GSE101585).

\section{Authors' contributions}

GH, WY and JL designed the study and conducted the experiments. CY and YX contributed to the data acquisition and collection. YW, JW and MC were responsible for analyzing and interpreting the data. GH, WY and JL drafted the manuscript. All authors read and approved the final manuscript.

\section{Ethics approval and consent to participate}

All procedures in the present study were approved by the Ethics Committee of the Liaocheng People's Hospital.

\section{Patient consent for publication}

Not applicable.

\section{Competing interests}

The authors declare that they have no competing interests.

\section{References}

1. Halliday BP, Baksi AJ, Gulati A, Ali A, Newsome S, Izgi C, Arzanauskaite M, Lota A, Tayal U, Vassiliou VS, et al: Outcome in dilated cardiomyopathy related to the extent, location, and pattern of late gadolinium enhancement. JACC Cardiovasc Imaging: Sep 6, 2018 (Epub ahead of print). doi: 10.1016/j.jcmg.2018.07.015.

2. Salerno M and Robinson AA: Risk stratification in nonischemic dilated cardiomyopathy in the era of personalized medicine: Can cardiac magnetic resonance with late gadolinium imaging 'enhance' our strategy? JACC Cardiovasc Imaging 11: 1285-1287, 2018.

3. Yancy CW, Jessup M, Bozkurt B, Butler J, Casey DE Jr, Colvin MM, Drazner MH, Filippatos GS, Fonarow GC, Givertz MM, et al 2017 ACC/AHA/HFSA Focused Update of the 2013 ACCF/AHA Guideline for the Management of Heart Failure: A Report of the American College of Cardiology/American Heart Association Task Force on Clinical Practice Guidelines and the Heart Failure Society of America. Circulation 136: e137-e161, 2017.

4. Garfinkel AC, Seidman JG and Seidman CE: Genetic pathogenesis of hypertrophic and dilated cardiomyopathy. Heart Fail Clin 14 139-146, 2018.

5. Harakalova M and Asselbergs FW: Systems analysis of dilated cardiomyopathy in the next generation sequencing era. Wiley Interdiscip Rev Syst Biol Med 10: e1419, 2018.

6. Merlo M, Cannatà A, Gobbo M, Stolfo D, Elliott PM and Sinagra G: Evolving concepts in dilated cardiomyopathy. Eur J Heart Fail 20: 228-239, 2018.

7. Thum T and Condorelli G: Long noncoding RNAs and microRNAs in cardiovascular pathophysiology. Circ Res 116: 751-762, 2015.
8. CabiliMN,TrapnellC,Goff L,Koziol M,Tazon-Vega B,Regev A and Rinn JL: Integrative annotation of human large intergenic noncoding RNAsreveals global properties and specific subclasses.Genes Dev 25: 1915-1927, 2011.

9. Eades G, Zhang YS, Li QL, Xia JX, Yao Y and Zhou Q: Long non-coding RNAs in stem cells and cancer. World J Clin Oncol 5: 134-141, 2014.

10. Zhang Y, Li Y, Wang J and Lei P: Long noncoding RNA ferritin heavy polypeptide 1 pseudogene 3 controls glioma cell proliferation and apoptosis via regulation of the microRNA-224-5p/tumor protein D52 axis. Mol Med Rep 18: 4239-4246, 2018.

11. Lorenzen JM, Martino F and Thum T: Epigenetic modifications in cardiovascular disease. Basic Res Cardiol 107: 245, 2012.

12. Ounzain S, Crippa S and Pedrazzini T: Small and long non-coding RNAs in cardiac homeostasis and regeneration. Biochim Biophys Acta 1833: 923-933, 2013.

13. Calore M, De Windt LJ and Rampazzo A: Genetics meets epigenetics: Genetic variants that modulate noncoding RNA in cardiovascular diseases. J Mol Cell Cardiol 89: 27-34, 2015.

14. Xie Z, Xia W and Hou M: Long intergenic noncoding RNA-p21 mediates cardiac senescence via the $\mathrm{Wnt} / \beta$-catenin signaling pathway in doxorubicin-induced cardiotoxicity. Mol Med Rep 17: 2695-2704, 2018

15. Yang KC, Yamada KA, Patel AY, Topkara VK, George I, Cheema FH, Ewald GA, Mann DL and Nerbonne JM: Deep RNA sequencing reveals dynamic regulation of myocardial noncoding RNAs in failing human heart and remodeling with mechanical circulatory support. Circulation 129: 1009-1021, 2014.

16. Huang ZP, Ding Y, Chen J, Wu G, Kataoka M, Hu Y, Yang JH, Liu J, Drakos SG, Selzman CH, et al: Long non-coding RNAs link extracellular matrix gene expression to ischemic cardiomyopathy. Cardiovasc Res 112: 543-554, 2016.

17. Liu R, Liao X, Li X, Wei H, Liang Q, Zhang Z, Yin M, Zeng X, Liang $\mathrm{Z}$ and $\mathrm{Hu} \mathrm{C}$ : Expression profiles of long noncoding RNAs and mRNAs in post-cardiac arrest rat brains. Mol Med Rep 17: 6413-6424, 2018

18. Taichman DB, McGoon MD, Harhay MO, Archer-Chicko C, Sager JS, Murugappan M, Chakinali MM, Palevsky HI and Gallop R: Wide variation in clinicians' assessment of New York Heart Association/World Health Organization functional class in patients with pulmonary arterial hypertension. Mayo Clin Proc 84: 586-592, 2009.

19. Maron BJ, Towbin JA, Thiene G, Antzelevitch C, Corrado D, Arnett D, Moss AJ, Seidman CE and Young JB; American Heart Association; Council on ClinicalCardiology, Heart Failure and Transplantation Committee; Quality of Care and Outcomes Research and Functional Genomics and Translational BiologyInterdisciplinary Working Groups; Council on Epidemiology and Prevention: Contemporary definitions and classification of the cardiomyopathies: An American Heart Association Scientific Statement from the Council on Clinical Cardiology, Heart Failure and Transplantation Committee; Quality of Care and Outcomes Research and Functional Genomics and Translational Biology Interdisciplinary Working Groups; and Council on Epidemiology and Prevention. Circulation 113: 1807-1816, 2006.

20. WRITING COMMITTEE MEMBERS, Yancy CW, Jessup M, Bozkurt B, Butler J, Casey DE Jr, Drazner MH, Fonarow GC, Geraci SA, Horwich T, et al: 2013 ACCF/AHA guideline for the management of heart failure: A report of the American College of Cardiology Foundation/American Heart Association Task Force on practice guidelines. Circulation 128: e240-e327, 2013.

21. Livak KJ and Schmittgen TD: Analysis of relative gene expression data using real-time quantitative PCR and the 2(-Delta Delta C(T)) method. Methods 25: 402-408, 2001.

22. Yang W, Han Y, Yang C, Chen Y, Zhao W, Su X, Yang K and Jin W: MicroRNA-19b-1 reverses ischaemia-induced heart failure by inhibiting cardiomyocyte apoptosis and targeting Bcl2 111/BIM. Heart Vessels: Jan 3, 2019 (Epub ahead of print). doi: 10.1007/s00380-018-01336-3.

23. Haeussler M, Zweig AS, Tyner C, Speir ML, Rosenbloom KR, Raney BJ, Lee CM, Lee BT, Hinrichs AS, Gonzalez JN, et al: The UCSC Genome Browser database: 2019 update. Nucleic Acids Res 47: D853-D858, 2019.

24. Kent WJ: BLAT-the BLAST-like alignment tool. Genome Res 12: 656-664, 2002. 
25. Huang da W, Sherman BT and Lempicki RA: Systematic and integrative analysis of large gene lists using DAVID bioinformatics resources. Nat Protoc 4: 44-57, 2009.

26. Fatica A and Bozzoni I: Long non-coding RNAs: New players in cell differentiation and development. Nat Rev Genet 15: 7-21, 2014

27. Yang N, Chen J, Zhang H, Wang X, Yao H, Peng Y and Zhang W: lncRNA OIP5-AS1 loss-induced microRNA-410 accumulation regulates cell proliferation and apoptosis by targeting KLF10 via activating PTEN/PI3K/AKT pathway in multiple myeloma. Cell Death Dis 8: e2975, 2017.

28. Cajigas I, Leib DE, Cochrane J, Luo H, Swyter KR, Chen S, Clark BS, Thompson J, Yates JR III, Kingston RE and Kohtz JD: Evf2 lncRNA/BRG1/DLX1 interactions reveal RNA-dependent inhibition of chromatin remodeling. Development 142: 2641-2652, 2015.

29. Wang R, Shi Y, Chen L, Jiang Y, Mao C, Yan B, Liu S, Shan B, Tao Y and Wang $\mathrm{X}$ : The ratio of FoxA1 to FoxA2 in lung adenocarcinoma is regulated bylncRNAHOTAIR and chromatinremodelingfactorLSH. Sci Rep 5: 17826, 2015.

30. Devaux Y, Zangrando J, Schroen B, Creemers EE Pedrazzini T, Chang CP, Dorn GW II, Thum T and Heymans S; Cardiolinc network: Long noncoding RNAs in cardiac development and ageing. Nat Rev Cardiol 12: 415-425, 2015.

31. Gao L, Liu Y, Guo S, Yao R, Wu L, Xiao L, Wang Z, Liu Y and Zhang Y: Circulating long noncoding RNA HOTAIR is an essential mediator of acute myocardial infarction. Cell Physiol Biochem 44: 1497-1508, 2017.

32. Zhang Y,Zhang M, Xu W, Chen J and Zhou X: The long non-coding RNA H19 promotes cardiomyocyte apoptosis in dilated cardiomyopathy. Oncotarget 8: 28588-28594, 2017.

33. Wang K, Long B, Zhou LY, Liu F, Zhou QY, Liu CY, Fan YY and Li PF: CARL IncRNA inhibits anoxia-induced mitochondrial fission and apoptosis in cardiomyocytes by impairing miR-539-dependent PHB2 downregulation. Nat Commun 5: 3596,2014
34. Ounzain S, Micheletti R, Beckmann T, Schroen B, Alexanian M, Pezzuto I, Crippa S, Nemir M, Sarre A, Johnson R, et al: Genome-wide profiling of the cardiac transcriptome after myocardial infarction identifies novel heart-specific long non-coding RNAs. Eur Heart J 36: 353-368a, 2015.

35. Fatkin D, Seidman CE and Seidman JG: Genetics and disease of ventricular muscle. Cold Spring Harb Perspect Med 4: a021063, 2014.

36. Burke MA, Cook SA, Seidman JG and Seidman CE: Clinical and mechanistic insights into the genetics of cardiomyopathy. J Am Coll Cardiol 68: 2871-2886, 2016.

37. Herman DS, Lam L, Taylor MR, Wang L, Teekakirikul P, Christodoulou D, Conner L, DePalma SR, McDonough B, Sparks E, et al: Truncations of titin causing dilated cardiomyopathy. N Eng J Med 366: 619-628, 2012.

38. Muelas N, Hackman P, Luque H, Garcés-Sánchez M, Azorín I, Suominen T, Sevilla T, Mayordomo F, Gómez L, Martí P, et al: MYH7 gene tail mutation causing myopathic profiles beyond Laing distal myopathy. Neurology 75: 732-741, 2010.

39. Eilebrecht S, Benecke BJ and Benecke AG: Latent HIV-1 TAR regulates $7 \mathrm{SK}$-responsive $\mathrm{P}-\mathrm{TEFb}$ target genes and targets cellular immune responses in the absence of Tat. Genomics Proteomics Bioinformatics 15: 313-323, 2017.

(i) $\Theta$ This work is licensed under a Creative Commons Attribution-NonCommercial-NoDerivatives 4.0 International (CC BY-NC-ND 4.0) License. 